\title{
Phytoremediation of Indoor Air
}

\author{
Creighton K. Thomas \\ Department of Materials Science and Engineering, Northwestern University, \\ Evanston, IL 60208
}

\author{
Kwang Jin Kim \\ Urban Agriculture Research Division, National Institute of Horticultural and \\ Herbal Science, Rural Development Administration, Suwon 441-440, Korea
}

\author{
Stanley J. Kays ${ }^{1}$ \\ Department of Horticulture, Plant Sciences Building, The University of \\ Georgia, Athens, GA 30602
}

Additional index words. air quality, volatile organic compounds, VOC, health hazard, mathematical model, effect of indoor plants, potential of phytoremediation

\begin{abstract}
Volatile organic compounds (VOCs) in homes and offices represent a potentially serious health problem for exposed individuals. While certain indoor plants have been shown to remove VOCs in small test chambers, the results have not consistently translated to larger, more complex structures. We report the results of a mathematical model that assesses the effect of plants on the removal of benzene or other VOCs in buildings, incorporating the primary variables modulating indoor air VOC concentration. Building air volume, amount of plant material, VOC concentration and air exchange, VOC emanation, and plant phytoremediation rates can be altered over ranges reported in the literature, clarifying the relationship among these parameters and thereby identifying the most appropriate interior air remediation options. The results indicate existing published phytoremediation rates determined using small test chambers are far higher than can be achieved with static potted plants in buildings, and facilitated air movement through the plant media will most likely be essential for phytoremediation to be a viable means of improving indoor air quality.
\end{abstract}

A dramatic increase in human health maladies has coincided with our exposure to an increasingly complex chemical environment (Genuis, 2012; Mostafalou and Abdollihi, 2013; Prüss-Ustün et al., 2011). Volatile organic compounds (VOCs) represent a category of environmental chemicals that are a major contributor to reduced indoor air quality. Humans inhale a diverse array of VOCs from the air in homes and offices. Some VOCs are known to be toxic, thus their prevalence poses a serious health hazard (Stacey and Winder, 2004). This effect is exacerbated in technologically advanced countries where people often spend more than $90 \%$ of their time indoors (Jenkins et al., 1992). Removing VOCs from indoor air is therefore a vital public health concern.

Methods for VOC removal vary widely in cost and effectiveness. Cutting-edge physical and chemical means of removing VOCs via adsorption, photocatalysis, or reaction with ozone (Carslaw, 2007; Mo et al., 2009; Steinberg et al., 2005) are limited practically by high initial and operational costs and by their dependence on heating/cooling systems to function properly. A simpler biological method, VOC removal by indoor plants, does not suffer from any of these drawbacks:

Received for publication 21 Jan. 2015. Accepted for publication 20 Mar. 2015.

${ }^{1}$ To whom reprint requests should be addressed; e-mail kaysstan@uga.edu. startup and maintenance costs are minimal, no specialized equipment, electricity, or other resources are necessary, and plants can continually remove VOCs, day and night. As part of the manned space program, NASA scientists in the 1980s found that certain indoor plant species and their associated microorganisms effectively removed VOCs in closed systems, purifying the air (Wolverton, 1986). But when indoor plants were tested in situ for their ability to remove VOCs in buildings, the results have ranged from modest to no effect (Apte and Apte, 2010; Kim et al., 2009; Pegas et al., 2012), leaving open the question, whether plants actually represent a viable solution for modulating the concentration of VOCs in a home or office environment. Buildings, in contrast to small sealed chambers, are much more complex systems, often having significant air exchange with the exterior and varying rates of VOC emanation from interior sources, neither of which have been measured in existing in situ studies. Here, we report the results of a mathematical model that assesses the effect of plants on removal of VOCs in buildings.

To model removal of VOCs from buildings by potted plants and their associated microbes, we describe the concentration $\rho$ of a single VOC (e.g., benzene) as a function of the relevant experimental parameters. While there may be some difference in removal rate between one and several gases, we assume here that different VOCs may be described independently using the same model, albeit with different values for parameters such as VOC emanation rate, VOC removal rate by plants, and initial concentration of the VOC. We consider the range of values seen in the literature as inputs to the model. To evaluate the efficacy of plant removal for a particular set of parameters, we compare the steadystate VOC concentrations of two systems that are identical except that one contains plants and the other does not. We also evaluate the speed at which plants can be expected to improve the conditions to ensure that it occurs on a useful timescale. Finally, we reanalyze some results from the literature in light of our model and recommend methods of data collection and reporting that would be most useful for a continuing improvement in the understanding of phytoremediation.

\section{Mathematical Description}

We consider a building of total volume $V$ that exchanges air with the outside world at rate $r_{\mathrm{x}}$, with units of volume per time. This exchange could be due to direct openings such as cracks, windows, or doors, or it could be due to a powered ventilation system. Especially in closed buildings, it might also be necessary to consider diffusion through the walls as a contribution to air exchange. We assume that the air inside this building is well-mixed so that complicated fluid dynamics equations are unnecessary to describe the system. Significant air flow inside the building, such as from a ventilation system, will increase this tendency, which can otherwise be justified empirically. The outside air is presumed to have negligible VOC concentrations compared with the inside air. After normalizing for building volume, the air exchange will tend to decrease the VOC concentration at a rate given by $r_{\mathrm{x}} \rho / V$, where $\rho=$ VOC concentration in $\mathrm{mg} \cdot \mathrm{m}^{-3}$.

Inside the building, some mass of a VOC emanates into the air from a source (or several sources) at rate $r_{\mathrm{em}}$, with units of VOC mass per time. This emanation will tend to increase the VOC concentration at a rate given by $r_{\mathrm{em}} / V$. Emanation rates are often measured in terms of concentration, so that the units for most measurements in the literature are those of $r_{\mathrm{em}} / V$. Some experiments have involved injection of a VOC into a chamber quickly to measure the decay in its concentration. In this case, the VOC concentration is an initial condition and $r_{\mathrm{em}}$ will be zero. In general, $r_{\mathrm{em}}$ could vary with time because of some daily or yearly cycle, depletion of the source, or addition of new sources. We will not consider this time dependence here, but it does not affect the underlying model, and it is straightforward to obtain a numerical solution of this more general case.

If plants (and their associated microbes) are present, they will tend to decrease the VOC concentration in the building. This removal process could be rate-limited, where the instantaneous VOC removal rate for the plants is independent of concentration, or it could be concentration-limited, where the 
removal rate is proportional to the concentration. A priori, both are plausible scenarios, but we find that the rate-limited case typically produces unphysical results for experimentally relevant parameters, so we focus on the concentration-limited case. In this case, the plants lower the VOC mass with rate given by $\alpha A_{\mathrm{p}} \rho$, with $\alpha$ being a proportionality constant to be extracted by fitting to data, and $A_{\mathrm{p}}$ being the area of the plants. In particular, $\alpha$ allows direct conversion between plant area and the usable volume of building air to be processed by the plant in a given amount of time. The concentration therefore decreases with rate $\alpha A_{\mathrm{p}} \rho / V$. The proportionality constant $\alpha$ ends up being the characteristic parameter defining the removal properties of plants; it can be seen as a removal efficiency rate, with plants that have higher values of $\alpha$ being more effective at removing VOCs. Its units are the same as velocity. This arises because $\alpha$ represents the flux corresponding to a volume of air moving through the plant surface area.

The definition of $A_{\mathrm{p}}$ is intentionally left somewhat vague, as it may be treated as a general measure of plant mass with units of area. One might consider this to correspond to the total leaf area of the plants, assuming other measures of plant mass to scale with the leaf area. It would also be reasonable to focus on the cross-sectional area of the air coming into contact with microbes in the soil of the plants, or on some more complicated combination of these parameters. Discrepancies between these definitions may be absorbed into the constant $\alpha$, allowing for the comparison of data using different measures, but the typical convention of measuring total leaf area will allow for reasonably consistent results among similar experiments. The crucial feature of $A_{\mathrm{p}}$ in the model is that it has units of area and scales with the number of plants used.

With the three processes that alter VOC concentration defined, the differential equation describing the concentration of the VOC as a function of time may then be written as

$$
\mathrm{d} \rho=\left(r_{\mathrm{em}} / V-\alpha A_{\mathrm{p}} \rho / V-r_{\mathrm{x}} \rho / V\right) \mathrm{d} t .
$$

In the equation, $\mathrm{d} \rho$ and $\mathrm{d} t$ are differentials in VOC concentration and time, respectively. In general, this equation is straightforward to solve numerically. Furthermore, we may assume that the parameters $r_{\mathrm{em}}, A_{\mathrm{p}}$, and $r_{\mathrm{x}}$ do not vary with time. This assumption is justified so long as changes in these parameters are slow compared with the response of the system to the changes. If these parameters are all time-independent, the solution to the model is

$\rho(t)=\left(\rho_{0}-\rho_{\infty}\right) \exp \left[-\left(\alpha A_{\mathrm{p}}+r_{\mathrm{x}}\right) t / V\right]+\rho_{\infty}$

with $\rho_{0}$ being the concentration at time $t=0$, and $\rho_{\infty}=r_{\mathrm{em}} /\left(\alpha A_{\mathrm{p}}+r_{\mathrm{x}}\right)$ being the steadystate concentration. Note that $\rho_{\infty}$ does not depend on the volume of the building, although the speed of the approach to this steady state concentration does. The closed-structure case with no air exchange corresponds to the limit that $r_{\mathrm{x}} \rightarrow 0$, while the case of VOC removal by air exchange with no plants is the limit that $A_{\mathrm{p}} \rightarrow 0$.

To assess the impact of adding plants, we compare the steady-state concentration with plants of total area $A_{\mathrm{p}}, \rho_{\infty}\left(A_{\mathrm{p}}\right)$, to that of an otherwise identical system with no plants $\rho_{\infty}$ $\left(A_{\mathrm{p}} \rightarrow 0\right)$. The fraction $\phi$ of VOC removal from a building that can be attributed to the plants is given by

$$
\phi=\frac{\rho_{\infty}\left(A_{\mathrm{p}} \rightarrow 0\right)-\rho_{\infty}\left(A_{\mathrm{p}}\right)}{\rho_{\infty}\left(A_{\mathrm{p}} \rightarrow 0\right)}=\frac{1}{1+r_{x} /\left(\alpha A_{\mathrm{p}}\right)}
$$

so that $\phi$ indicates the effectiveness of the plants at removing VOCs: if $\phi$ is close to 1 , the plants account for most of the VOC removal, and if $\phi$ is close to zero, the plants are ineffective compared with air exchange. Experimentally, one might consider equilibrating the VOC concentration in a building with no plants, then adding plants and measuring the concentration as a function of time after this. In such an experiment, the decrease in concentration caused by the plants is given by

$$
\frac{\rho_{\infty}}{\rho_{0}}=\frac{1}{1+\alpha A_{\mathrm{p}} / r_{\mathrm{x}}}=1-\phi
$$

which gives a direct way to measure $\phi$ in a single experiment.

It is also of interest to compute how quickly the system approaches this steady state concentration, to ensure that this concentration is relevant at reasonable time scales. The half-life for the time-dependent (exponential decay) portion of the concentration is given by

$$
t_{1 / 2}=\frac{V \log (2)}{r_{\mathrm{x}}+\alpha A_{\mathrm{p}}}
$$

where $\log (2)$ is the natural logarithm of 2 . The half-life of the system with plants included is always smaller than the half-life of the exchange process without plants (which can be obtained by setting $A_{\mathrm{p}} \rightarrow 0$ in the above equation), thus plants can only speed up the approach to the steady state concentration, compared with a plant-free system with an identical air-exchange rate.

\section{Results}

Experimental removal rates for plants are typically measured as a concentration changes per time. The value of the parameter $\alpha$ can be extracted from such measurements if the concentration at which the measurement was made is known. Ideally, these would be extracted from curve fits to data for concentration as a function of time according to the equations for $\rho(t)$ given previously. Any of the relevant equations containing $\alpha$ could be used, but as one example, the half-life may be readily applied to extracting $\alpha$. In Kim et al. (2008), plants with total area $\approx 0.1 \mathrm{~m}^{2}$ were kept in a sealed $1-\mathrm{m}^{3}$ enclosure (so $r_{\mathrm{x}}=0$ ), and the half-life of the VOC decay is seen to be 1-2 h. The value for $\alpha$ can then be calculated as $\alpha=(V \log 2) /\left(A_{\mathrm{p}} t_{1 / 2}\right) \approx 3-7$ $\mathrm{m} \cdot \mathrm{h}^{-1}$. From this type of analysis, we have found that values for $\alpha$ from the data in the literature roughly lie in the range $0.1-10$ $\mathrm{m} \cdot \mathrm{h}^{-1}$ (Kim et al., 2008; Liu et al., 2007; Orwell et al., 2004; Tarran et al., 2007; Wood et al., 2002).

In the above example using the results of Kim et al. (2008), the experiment involved injecting the VOC once and measuring the decay in concentration, presumably with no additional VOC emanation. Larger buildings, on the other hand, may tend to have a continuous (nonzero) emanation of VOCs. Our model addresses both these cases, allowing for direct comparison among the setups. The injection-only case is slightly simpler to handle, because $r_{\mathrm{em}}=0$ as the experiment progresses, so $\rho_{\infty}=0$, making the half-life, and therefore $\alpha$ is easy to extract from the data. In the continuousemission case, one can extract $\alpha$ by fitting the data to the exponential decay equation for $\rho(t)$ with $\alpha$ and $r_{\mathrm{em}}$ as fit parameters (if $r_{\mathrm{em}}$ is unknown). If an accurate value of $\alpha$ is desired, it is important to either have a reasonable estimate of $r_{\mathrm{x}}$ or repeat the experiment with multiple values for $A_{\mathrm{p}}$, as $r_{\mathrm{x}}$ is not completely independent from $\alpha$ in the equations. More complicated time- or concentration-dependent emanation may also be handled by this model, although it will be necessary to solve the differential equation numerically in some cases.

The effect of plants and air exchange on VOC concentration is illustrated in Figure 1. All curves in this figure correspond to typical values for a home or office: $V=300 \mathrm{~m}, A_{\mathrm{p}}=$ $4.56 \mathrm{~m}^{2}$, with $\rho_{0}=1 \mathrm{ppm}=3.19 \mathrm{mg} \cdot \mathrm{m}^{-3}$, and $r_{\mathrm{em}}=3.19 \mathrm{mg} \cdot \mathrm{h}^{-1}$. This leaf area is considered roughly equivalent to four large floor and four table plants. [Dumb cane-Dieffenbachia seguine (Jacq.) Schottz (floor); Boston fern-Nephrolepis exaltata (L.) Schott, approximate combined total surface area (i.e., four plant/species) occupied $2.4 \mathrm{~m}^{2}$.] Two different exchange rates are used in Figure 1, $r_{\mathrm{x}}=0,30 \mathrm{~m}^{3} \cdot \mathrm{h}^{-1}$, and $\alpha$ is shown varying from 0.1 to $10 \mathrm{~m} \cdot \mathrm{h}^{-1}$, the range of values we have seen from data in the literature. If the values of $\alpha$ are high enough $\left(\approx 0.3\right.$ or $1 \mathrm{~m} \cdot \mathrm{h}^{-1}$ or higher), a significant reduction is seen in $\rho$ over the course of a few days, even with no air exchange (Fig. 1A). When air exchange is higher (Fig. 1B), there is already a significant reduction due to the air exchange alone. Some additional reduction, plus a speed up in the approach to the final concentration, may be achieved by adding plants in this case. But the plants clearly have a more modest comparative effect when the air-exchange rate is already high.

\section{Discussion}

The model shown here suggests that plants with moderate to high VOC removal rates should be effective at lowering VOC concentrations in buildings, even when some air exchange with the outside occurs. While quantities such as $\alpha$ may be used to describe both large and small buildings, the measured phytoremediation rates that are typically obtained in small test chambers $\left(\leq 1 \mathrm{~m}^{3}\right)$ 


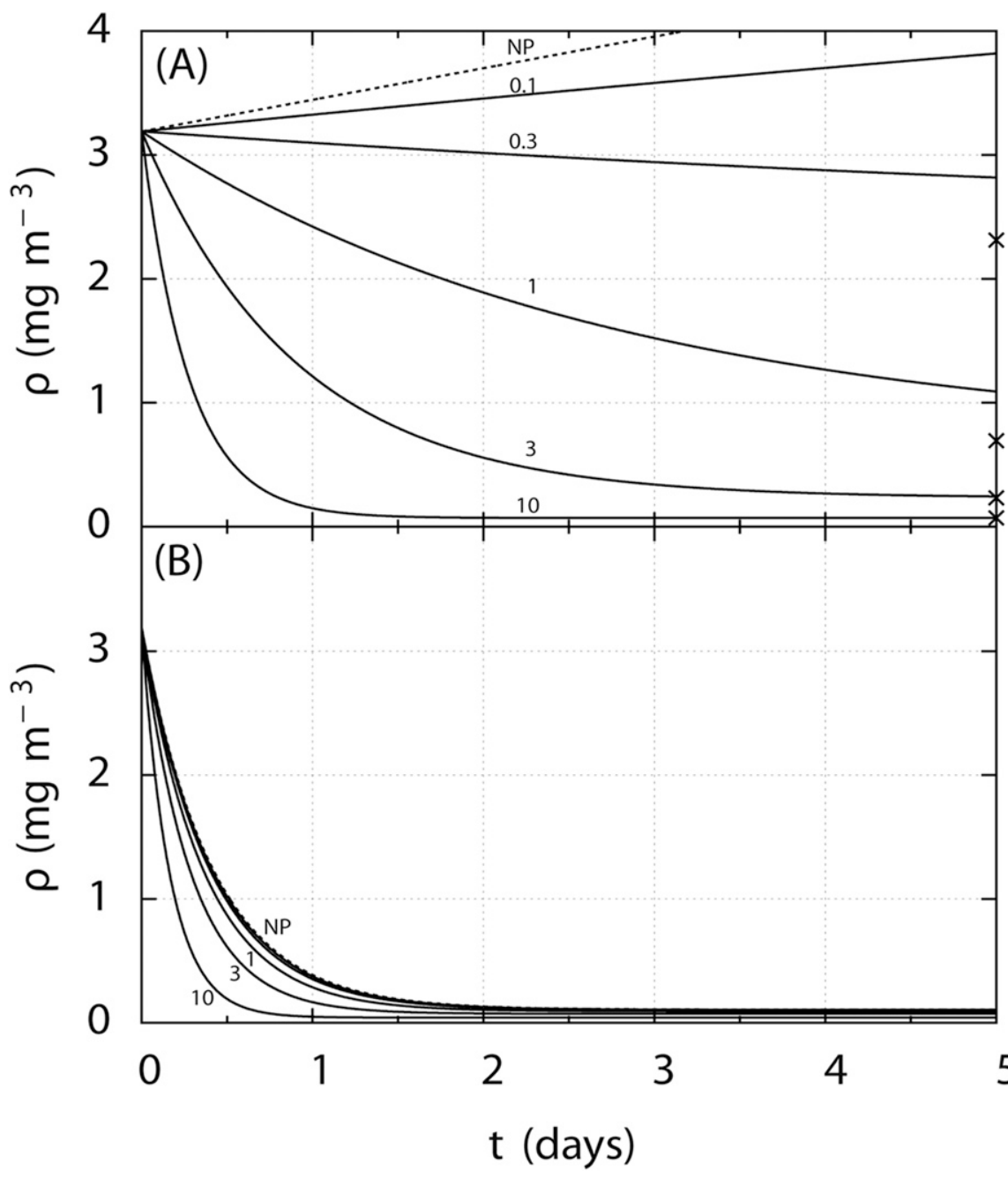

Fig. 1. Benzene concentration as a function of time for varying exchange rate $r_{\mathrm{x}}$ and plant volatile organic compounds (VOC) removal effectiveness $\alpha$. All curves correspond to $V=300 \mathrm{~m}^{3}, A_{\mathrm{p}}=4.56 \mathrm{~m}^{2}, \rho_{0}=1$ $\mathrm{ppm}=3.19 \mathrm{mg} \cdot \mathrm{m}^{-3}$, which are typical values for a home or office. (A) The exchange rate of air $r_{\mathrm{x}}=$ $0 \mathrm{~m}^{3} \cdot \mathrm{h}^{-1}$; the dashed line at the top is when no plants (NP) are present. Solid lines from top to bottom $\alpha$ $=0.1,0.3,1,3$, and 10. Larger values of $\alpha$ indicate that plants are more efficient at removing VOCs. The concentration decay in the $r_{\mathrm{x}}=0$ case is slow enough that the eventual steady state concentration is not apparent in all cases. The steady state concentration is marked by a multiplication sign $(\times)$ on the right axis; each multiplication sign is the final value for the nearest curve directly above it. (B) The air exchange rate $r_{\mathrm{x}}=30 \mathrm{~m}^{3} \cdot \mathrm{h}^{-1}$; the dashed line at the top is when NP are present. Solid lines from top to bottom $\alpha=0.1,0.3,1,3$, and 10 .

may not directly correspond to realistic building environments. When the phytoremediation rate was measured in sealed rooms $\left(60 \mathrm{~m}^{3}\right)$ and compared with that of small chambers $\left(1 \mathrm{~m}^{3}\right)$ under otherwise identical conditions, the VOC removal rate in the rooms was $1 / 20$ th of that of the small chambers (Kim et al., 2009). Based on this result, to obtain an equivalent reduction in VOC concentration (i.e., $67 \%$ ) in a $300-\mathrm{m}^{3}$ house, it would require an unrealistically large number of plants (i.e., 360 or 750 plants depending upon the species; Gardenia jasminoide J. Ellis vs. Rosmarinus officinalis L., respectively). We note that the half-life of VOC decay scales with the volume of the room, so larger rooms will not be able to remove VOCs as quickly, with all other parameters being equal. This is ameliorated if the number of plants also scales with the studies (Anon, 1992; Apte and Apte, 2010; Dingle et al., 2000; Lim et al., 2009).

The difference in experimental removal efficiency with increasing air volume could also be due to an inadequate rate of VOC diffusion to/into the plant and media, a position that is supported by the fact that facilitated diffusion substantially increases the rate of removal. For example, using a positive or negative pressure to move air through the pot significantly increases the VOC removal rate (Son and Choi, 2009; Wolverton and Wolverton, 1995) and with plant biofiltration walls (Darlington et al., 2001) where the air is circulated through a porous vertical wall of plants and microbe-containing root media, the removal rate is greatly increased compared with moving the air around potted plants. As the air flow through the plant biofilter increases, the amount of VOC removed increases. This suggests that the wellmixed assumption made in our mathematical model may not be satisfied in real-world applications. It is also possible that facilitated VOC diffusion actually increases the effectiveness of VOC removal by plants, which would alter the value of $\alpha$. Increasing the ventilation in the room could dramatically lower VOC levels, especially if the ventilation system mimics these biofiltration walls.

The increased efficiency achieved by passing air through the media underscores the importance of soil microorganisms that remove VOCs and do so both day and night. The ratio of formaldehyde removed by aerial plant parts vs. the root-zone was $\approx 1: 1$ during the day but declined to $1: 11$ at night when the stomata were closed (Kim et al., 2009). Wolverton and Wolverton (1993) found that the aerial plant parts to media VOC removal ratio also varied with plant species and the VOC in question. During the day, Dieffenbachia sequine (Jacq.) Schott var. seguine (syn. D. maculate) and Nephrolepis exaltata (L.) Schott had similar ratios $(\approx 1: 1$ aerial plant parts: root zone) for xylene while ratios for formaldehyde favored the root zone (37:63 Diffenbachia sp. to 40:60 Aglaonema $\mathrm{sp}$.). Bacteria associated with the roots are the primary microorganisms responsible for removing VOCs. Recently, isolates of eight bacteria species that have the ability to live on toluene as their sole carbon source were identified (Zhang et al., 2013).

Secondary causes for the elevated phytoremediation rates in small chambers include inappropriate experimental methods that favor removal. Examples are the use of fans or pumps to facilitate air movement in the chamber, higher light intensity than commonly found in homes and offices, the lack of sufficient plant acclimatization to indoor conditions, and the effect of reduced $\mathrm{CO}_{2}$ concentration on stomatal aperture.

Using individual or a small number of plants within individual pots is far more likely to be accepted for use in homes and offices than plant biofiltration walls or large filtration systems (Steinberg et al., 2005) in that the former is far less expensive, does not require professional installation and maintenance, and 
allows more aesthetically pleasing positioning of the plants around the building, among other advantages. Our results indicate that facilitated movement of the air through the pot containing plant(s) is most likely essential for obtaining adequate VOC reduction with a reasonable number of plants in a home or office; however, it entails the use of electricity that limits where phytoremediation can be used and increases the expense.

A confounding factor in studying the phytoremediation of indoor air is the numerous plant, microbe, and building variables. Phytoremediation involves a highly complex system; one in which the alteration in any one of a large number of variables can have a significant impact on others and in turn, on VOC removal. As a consequence, each structure represents a unique situation requiring its own remediation solution.

Individual structures vary in size, composition, amount of air exchange, number and kind of inhabitants, geographical location, and a myriad of other ways that result in the VOC composition and concentration of the air being highly variable among buildings. Indoor plants also vary widely in phytoremediation rate, the VOCs they effectively remove and other biological factors. In addition, little is known about the microbe population that varies in composition, size, sensitivity to VOCs, and the effect of plant species, media, temperature, wetting/drying cycles, and other variables. At present, these biological variables and their interactions are not well understood.

The model described here incorporates this enormous variability in terms of four primary parameters $\left(r_{\mathrm{em}}, \alpha, A_{\mathrm{p}}\right.$, and $\left.r_{\mathrm{x}}\right)$ that control indoor-air VOC concentrations. Given appropriate estimates of these variables to describe a given system, this model may be used to determine which remediation option is superior (e.g., increased air exchange, using plants, identifying and removing the VOC source). With an accurate estimate of the phytoremediation removal parameter $\alpha$, and knowing the air exchange and VOC emanation rates, the model also can provide fine tuning recommendations for the number of plants of selected species that would be required to reduce the concentrations of certain VOCs by a given percentage over a fixed period of time.

\section{Literature Cited}

Anon. 1992. Can plants help clean up the indoor air? Healthy Build. Intern. Mag. 2(1):10-11.

Apte, M.G. and J.S. Apte. 2010. A pilot study of the effectiveness of indoor plants for removal of volatile organic compounds in indoor air in a seven-story office building. Rept. Energy Analysis Environ. Impacts Dept., Lawrence Berkeley Nat. Lab, Berkeley, CA.

Carslaw, N. 2007. A new detailed chemical model for indoor air pollution. Atmos. Environ. 41:1164-1179.

Darlington, A.B., J.F. Dat, and M.A. Dixon. 2001. The biofiltration of indoor air: Air flux and temperature influences the removal of toluene, ethylbenzene, and xylene. Environ. Sci. Technol. 35:240-246.

Dingle, P., P. Tapsell, and S. Hu. 2000. Reducing formaldehyde exposure in office environments using plants. Bull. Environ. Contam. Toxicol. 64:302-308.

Genuis, S.J. 2012. What's out there making us sick. J. Environ. Public Health 2012:605137.

Jenkins, P.L., T.J. Phillips, E.J. Mulberg, and S.P. Hui. 1992. Activity patterns of Californians: Use of and proximity to indoor pollutant sources. Atmos. Environ. 26:2141-2148.

Kim, H.-H., J.-Y. Lee, J.-Y. Yang, K.-J. Kim, Y.-J. Lee, D.-C. Shin, and Y.-M. Lim. 2011. Evaluation of indoor air quality and health related parameters in office buildings with or without indoor plants. J. Jpn. Soc. Hort. Sci. 80:96-102.

Kim, K.J., M.J. Kil, M.I. Jeong, H.D. Kim, E.H. Yoo, S.J. Jeong, C.H. Pak, and K.C. Son. 2009. Determination of the efficiency of formaldehyde removal according to the percentage volume of pot plants occupying a room. Kor. Hort. Sci. Technol. 27:305-311.

Kim, K.J., M.J. Kil, J.S. Song, E.H. Yoo, K.C. Son, and S.J. Kays. 2008. Efficiency of volatile formaldehyde removal by indoor plants: Contribution of aerial plant parts versus the root zone. J. Amer. Soc. Hort. Sci. 133:1-6.

Lim, Y.-W., H.-H. Kim, J.-Y. Yang, K.-J. Kim, J.-Y. Lee, and D.-C. Shin. 2009. Improvement of indoor air quality by houseplants in new-built apartment buildings. J. Jpn. Soc. Hort. Sci. 78:456-462.

Liu, Y.J., Y.J. Mu, Y.G. Zhu, H. Ding, and N.C. Arens. 2007. Which ornamental plant species effectively remove benzene from indoor air? Atmos. Environ. 41:650-654.

Mo, J., Y. Zhang, and R. Yang. 2005. Novel insight into VOC removal performance of photocatalytic oxidation reactors. Indoor Air 15:291300.

Mostafalou, S. and M. Abdollahi. 2013. Pesticides and human chronic diseases: Evidences, mech- anisms, and perspectives. Toxicol. Appl. Pharmacol. 268:157-177.

Orwell, R.L., R.L. Wood, J. Tarran, F. Torpy, and M.D. Burchett. 2004. Removal of benzene by the indoor plant/substrate microcosm and implications for air quality. Water Air Soil Pollut. 157:193-207.

Pegas, P.N., C.A. Alves, T. Nunes, E.F. Bate-Epey, M. Evtyugina, and C.A. Pio. 2012. Could houseplants improve indoor air quality in schools? Toxicol. Environ. Health. Part A. 75 (22-23):1371-1380.

Prüss-Ustün, A., C. Vickers, P. Haeflinger, and R. Bertollini. 2011. Knowns and unknowns on burden of disease due to chemicals: A systematic review. Environ. Health. 10:9.

Son, K.-C. and J. Choi. 2009. Eco-friendly air purifier green plants, p. 82-83. In: Horticultural Wellbeing. Joongang Life Pub., Seoul, Korea. (in Korean.)

Stacey, N.H. and C. Winder. 2004. Toxicity of organic solvents, p. 364-389. In: Winder, C. and N.H. Stacey (eds.). Occupational toxicology. CRC Press, Boca Raton, FL.

Steinbury, I., C. Rohde, A. Bockreis, and J. Jager. 2005. Increase of the purification efficiency of biofilters by the use of complementary ionization step. Waste Mgt. 25(4):375-381.

Tarran, J., F. Torpy, and M. Burchett. 2007. Use of living pot-plants to cleanse indoor air-Research review. Proc. Sixth Intern. Conf. on Indoor Air Quality, Ventilation \& Energy Conservation in Buildings-Sustainable Built Environment, Sendai, Japan.

Wolverton, B.C. 1986. Houseplants, indoor air pollutants, and allergic reactions. National Space Technology Laboratories, NASA, Stennis Space Center, Bay St. Louis, MS.

Wolverton, B.C. and J.D. Wolverton. 1993. Plants and soil microorganisms: Removal of formaldehyde, xylene, and ammonia from the indoor environment. J. Mississippi Acad. Sci. 38:11-15.

Wolverton, B.C. and J.D. Wolverton. 1995. Indoor humidification and purification. U.S. Patent 5,433,923 A.

Wood, R.A., R. Orwell, J. Tarran, F. Torpy, and M.D. Burchett. 2002. Potted plant-growth media: interactions and capacities in removal of volatiles from indoor air. J. Environ. Hort. Biotechnol. 77:120-129.

Zhang, H., S.V. Svoboda, S.J. Kays, and M.Y. Habteselassi. 2013. Isolation and identification of toluene-metabolizing bacteria from rhizospheres of two indoor plants. Water Air Soil Pollution 224:1648. 